\title{
Added Value of Biparametric MRI and TRUS-Guided Systematic Biopsies to Clinical Parameters in Predicting Adverse Pathology in Prostate Cancer
}

This article was published in the following Dove Press journal: Cancer Management and Research

\author{
Hailang Liu' \\ Kun Tang' \\ Ding Xia' \\ Xinguang Wang' \\ Wei Zhu' \\ Liang Wang ${ }^{2}$ \\ Weimin Yang ${ }^{\prime}$ \\ Ejun Peng' \\ Zhiqiang Chen' \\ 'Department of Urology, Tongji Hospital, \\ Tongji Medical College, Huazhong \\ University of Science and Technology, \\ Wuhan 430030, Hubei, People's Republic \\ of China; ${ }^{2}$ Department of Radiology, \\ Tongji Hospital, Tongji Medical College, \\ Huazhong University of Science and \\ Technology, Wuhan 430030, Hubei, \\ People's Republic of China
}

Correspondence: Ejun Peng; Zhiqiang

Chen

Email qingyangfeng60@gmail.com; d201981784@hust.edu.cn
Objective: To develop novel models for predicting extracapsular extension (EPE), seminal vesicle invasion (SVI), or upgrading in prostate cancer $(\mathrm{PCa})$ patients using clinical parameters, biparametric magnetic resonance imaging (bp-MRI), and transrectal ultrasonography (TRUS)-guided systematic biopsies.

Patients and Methods: We retrospectively collected data from PCa patients who underwent standard (12-core) systematic biopsy and radical prostatectomy. To develop predictive models, the following variables were included in multivariable logistic regression analyses: total prostatespecific antigen (TPSA), central transition zone volume (CTZV), prostate-specific antigen (PSAD), maximum diameter of the index lesion at bp-MRI, EPE at bp-MRI, SVI at bp-MRI, biopsy Gleason grade group, and number of positive biopsy cores. Three risk calculators were built based on the coefficients of the logit function. The area under the curve (AUC) was applied to determine the models with the highest discrimination. Decision curve analyses (DCAs) were performed to evaluate the net benefit of each risk calculator.

Results: A total of 222 patients were included in this study. Overall, 83 (37.4\%), 75 (33.8\%), and 107 (48.2\%) patients had EPE, SVI, and upgrading at final pathology, respectively. The addition of bp-MRI data improved the discrimination of models for predicting SVI (0.807 vs 0.816 ) and upgrading ( 0.548 vs 0.625 ) but not EPE ( 0.766 vs 0.763 ). Similarly, models including clinical parameters, bp-MRI data, and information on systematic biopsies achieved the highest AUC in the prediction of EPE (0.842), SVI (0.913), and upgrading (0.794), and the three corresponding risk calculators yielded the highest net benefit.

Conclusion: We developed three easy-to-use risk calculators for the prediction of adverse pathological features based on patient clinical parameters, bp-MRI data, and information on systematic biopsies. This may be greatly beneficial to urologists in the decision-making process for PCa patients.

Keywords: prostate cancer, extracapsular extension, seminal vesicle invasion, upgrading, biparametric MRI, systematic biopsy, predictive model

\section{Introduction}

Prostate cancer $(\mathrm{PCa})$ is the most frequently diagnosed cancer among men, with 1,276,100 newly diagnosed cases in 2018 worldwide and 174,650 newly diagnosed cases in 2019 in the United States. ${ }^{1,2}$ It is the second leading cause of cancer-related death in male patients in the United States. ${ }^{2}$ More recently, the incidence and mortality of PCa have exhibited increasing trends in China. ${ }^{3}$ For the management of $\mathrm{PCa}$, it is of pivotal importance to identify $\mathrm{PCa}$ patients at a higher risk of extracapsular extension (EPE), seminal vesicle invasion (SVI), and upgrading at 
radical prostatectomy (RP). Preoperative knowledge of adverse pathological outcomes may inform more appropriate treatment options such as the use of modified surgical techniques and transition from surgical management to multimodality therapy. ${ }^{4}$

Prediction models that combine serum PSA levels, clinical stage, and Gleason grade in the biopsy specimens such as the Memorial Sloan Kettering Cancer Center (MSKCC) nomograms and the Partin tables are commonly used in clinical practice to predict the pathological stage of $\mathrm{PCa}$ and thus aid in preoperative decision-making. ${ }^{5-7}$ Furthermore, multiparametric magnetic resonance imaging (mp-MRI) has emerged as an important tool for detecting PCa patients with a higher risk of adverse pathology. ${ }^{8,9}$ Despite the moderate sensitivity of this imaging modality in predicting ECE and SVI, robust evidence suggests that the inclusion of information derived from mp-MRI or MRI-targeted biopsies improves the accuracy of models predicting adverse pathology for PCa patients. ${ }^{7,10-12}$ However, mp-MRI is expensive and time-consuming, and the high prevalence of PCa mp-MRI poses a significant financial burden on society. ${ }^{13,14}$ Interestingly, biparametric MRI (bp-MRI), consisting of T2-weighted imaging and diffusion-weighted imaging without dynamic contrastenhanced imaging, has shown comparable efficacy in detecting clinically significant PCa by mp-MRI. ${ }^{13,15}$ It could be speculated that bp-MRI may also have potential value in predicting adverse pathological features in $\mathrm{PCa}$ patients.

Therefore, we aim to develop easy-to-apply models designed for the prediction of EPE, SVI and upgrading at $\mathrm{RP}$, which integrates clinical parameters, information obtained at standard transrectal ultrasound (TRUS)guided biopsies, and bp-MRI findings. In addition, we aim to assess the performance of predictive models based on clinical parameters alone versus models including bpMRI and biopsy data in predicting adverse pathological outcomes.

\section{Patients and Methods}

\section{Patient Selection}

Patients who underwent radical prostatectomy at Tongji Hospital of Tongji Medical College, Huazhong University of Science and Technology between January 2016 and December 2019 were retrospectively enrolled in this study. This retrospective study was approved by the institutional review board (IRB) of Tongji Hospital, Tongji Medical
College, Huazhong University of Science and Technology. Because our study belonged to a retrospective study, patient consent to review their medical records were not required by the IRB. And all patient information was strictly confidential and our procedures were carried out according to the Declaration of Helsinki. Inclusion criteria: (1) prostate TRUS and bp-MRI performed for all patients before surgery; (2) standard systematic (12-core) TRUS-guided biopsy prior to surgery performed for all patients; (3) final pathological results of each patient involved detailed description of EPE, SVI, and Gleason grade group. Exclusion criteria: (1) neoadjuvant therapy prior to TRUS and MRI examination; (2) patients with incomplete clinical data; (3) unsatisfactory quality of MRI images. A bp-MRI was performed on all men using a 3 Tesla MRI scanner (MAGNETOM Skyra; Siemens, Medical Solutions, Erlangen, Germany). The bp-MRI findings were re-reported and scored by the same dedicated radiologist on a five-point scale using modified (no contrast-enhanced imaging) Prostate Imaging Reporting and Data System (PI-RADS) version 2 criteria. ${ }^{16}$ TRUS-guided biopsy was performed using a 2102 BK Ultrasound system (BK Medical A/S, Herlev, Denmark). This study finally enrolled 222 patients.

\section{Definition of Variables}

The clinical variables included patient age, total prostatespecific antigen (TPSA), prostate volume (PV), central transition zone volume (CTZV), prostate-specific antigen density (PSAD), maximum diameter of the index lesion at MRI, PI-RADS v2 score, clinical stage at digital rectal examination (DRE), EPE and SVI at MRI, global Gleason grade group (GG), number of positive biopsy cores, and final pathology results. Both the PV and CTZV were directly measured by TRUS and calculated using the formula for an ellipsoid. PSAD was defined as the ratio of TPSA to PV. The maximum diameter of the index lesion at MRI was derived from the lesion with the highest PIRADS v2 score or the one with the largest diameter among those lesions with the same PI-RADS v2 score. EPE and SVI at MRI were evaluated by the high spatial resolution T2-weighted images. ${ }^{17} \mathrm{GG}$ of the biopsy specimen was assigned following 2014 ISUP criteria. ${ }^{18}$ The global GG of the biopsy was defined as the most prevalent GG among all positive cores. Adverse pathological features of the present study were represented by EPE, SVI, and ISUP group upgrading at final pathology. The upgrades from biopsy to RP represented at least one grade difference in the GG. 


\section{Statistical Analysis}

Categorical variables were expressed as count (\%); however, nonparametric continuous variables were described using the median and interquartile range (IQR). Multivariable binary logistic regression analyses were performed to investigate the association of clinical parameters, bp-MRI findings and TRUS biopsy data with the prediction of adverse pathological features. First, we developed three novel risk calculators using clinical variables alone (TPSA, CTZV, and PSAD). Second, we further evaluated the utility of the risk calculator, which integrated clinical variables and bp-MRI information (maximum diameter of the index lesion at MRI, EPE at MRI and SVI at MRI). Finally, we assessed whether incorporating information obtained at TRUS-guided biopsies into the former models relied on clinical variables and bp-MRI information improved the identification of patients at high risk of adverse pathological features. The receiver-operating characteristic (ROC)-derived area under the curve (AUC) was applied to assess the discrimination of these prediction tools. Decision curve analysis (DCA) was conducted to determine the clinical net benefit associated with the use of the risk calculators at different threshold probabilities in the patient cohort. ${ }^{19}$ Statistical analyses were performed using IBM SPSS software (version 24.0; IBM Corp, Armonk, NY, USA) and R software (Version 3.6.0; https://www.R-project.org). $\mathrm{P}<0.05$ was considered statistically significant.

\section{Results}

\section{Baseline Patient Characteristics}

Descriptive characteristics for the overall patient cohort are shown in Table 1 . Of the 222 included patients, 83 (37.4\%) and 75 (33.8\%) patients had EPE and SVI at final pathology, respectively. Table 2 details the concordance between the biopsy global GG and the final RP GG, and the corresponding downgrades and upgrades for GG 1-5. The most prevalent GGs assigned on biopsy were GG 1 $(32.0 \%)$ and GG 4 (20.3\%). Overall, the disease was upgraded at final pathology in 107 patients $(48.2 \%)$. The median age at RP was 69 (IQR 64-76) years, and the median preoperative TPSA was 24.6 (IQR 11.6-49.4) ng/ $\mathrm{mL}$. In the overall cohort, the median maximum diameter of the index lesion at MRI was 2.0 (IQR 1.3-2.6) $\mathrm{cm}$, and $85(38.3 \%)$ patients had suspected EPE and $71(32.0 \%)$
Table I Descriptive Statistics of 222 Patients with Prostate Cancer Diagnosed with TRUS-Guided Biopsy and Treated with Radical Prostatectomy Between 2016 and 2019

\begin{tabular}{|c|c|}
\hline & Overall $(n=222)$ \\
\hline Age at surgery (yr), median (IQR) & $69(64-76)$ \\
\hline TPSA (ng/mL), median (IQR) & $24.6($ (II.6-49.4) \\
\hline fPSA (ng/mL), median (IQR) & $2.6(I . I-5.0)$ \\
\hline PV (mL), median (IQR) & $42.9(32.7-55.4)$ \\
\hline CTZV (mL), median (IQR) & $26.9(19.3-34.6)$ \\
\hline \multicolumn{2}{|l|}{ PSAD (ng/mL/mL), (n, \%) } \\
\hline$\leq 0.20$ & $34(15.3 \%)$ \\
\hline$>0.20$ & I 88 (84.7\%) \\
\hline \multicolumn{2}{|l|}{ Clinical T stage (\%) } \\
\hline $\mathrm{TI}$ & $26(11.7 \%)$ \\
\hline $\mathrm{T} 2$ & $128(57.7 \%)$ \\
\hline T3 & $63(28.4 \%)$ \\
\hline $\mathrm{T} 4$ & $5(2.2 \%)$ \\
\hline \multicolumn{2}{|l|}{ PI-RADS score (n, \%) } \\
\hline $1-2$ & $13(5.9 \%)$ \\
\hline 3 & $24(10.8 \%)$ \\
\hline 4 & $47(21.2 \%)$ \\
\hline 5 & $138(62.1 \%)$ \\
\hline Maximum diameter of the index lesion at bp- & $2.0(1.3-2.6)$ \\
\hline MRI (cm), median (IQR) & \\
\hline EPE at bp-MRI (n, \%) & $85(38.3 \%)$ \\
\hline SVI at bp-MRI (n, \%) & 71 (32.0\%) \\
\hline \multicolumn{2}{|l|}{ Biopsy grade group (n, \%) } \\
\hline I & $71(32.0 \%)$ \\
\hline 2 & $40(18.0 \%)$ \\
\hline 3 & 44 (19.8\%) \\
\hline 4 & $45(20.3 \%)$ \\
\hline 5 & $22(9.9 \%)$ \\
\hline No. of positive biopsy cores, median (IQR) & $6(3-11)$ \\
\hline $\begin{array}{l}\text { Linear percentage of tumor in total biopsy } \\
\text { cores }(\%) \text {, median (IQR) }\end{array}$ & $21.7(9.0-37.6)$ \\
\hline \multicolumn{2}{|l|}{ Gleason grade group at final pathology $(n, \%)$} \\
\hline 1 & $19(8.6 \%)$ \\
\hline 2 & $57(25.7 \%)$ \\
\hline 3 & $54(24.3 \%)$ \\
\hline 4 & 49 (22.1\%) \\
\hline 5 & $43(19.3 \%)$ \\
\hline PSM at final pathology (n, \%) & $99(44.6 \%)$ \\
\hline EPE at final pathology $(n, \%)$ & $83(37.4 \%)$ \\
\hline SVI at final pathology (n, \%) & $75(33.8 \%)$ \\
\hline
\end{tabular}

Abbreviations: TPSA, total prostate-specific antigen; fPSA, free prostate-specific antigen; PV, prostate volume; CTZV, central transitional zone volume; PSAD, prostate-specific antigen density; PI-RADS, Prostate Imaging Reporting and Data System; bP-MRI, biparametric MRI; EPE, extracapsular extension; SVI, seminal vesicle invasion; PSM, positive surgical margin. 
Table 2 Global Grade Groups on Biopsy and Radical Prostatectomy and Change in Grade

\begin{tabular}{|c|c|c|c|c|c|c|c|c|c|}
\hline \multirow[t]{3}{*}{ Biopsy GS (GG) } & \multirow[t]{3}{*}{$\mathbf{N}$} & \multicolumn{5}{|c|}{ GS (GG) at RP (N [\% of GS/GG]) } & \multirow{2}{*}{\multicolumn{3}{|c|}{ Change in Score (N [\% of GS/GG]) }} \\
\hline & & \multirow{2}{*}{$\begin{array}{l}6 \\
\text { (GGI) }\end{array}$} & \multirow{2}{*}{$\begin{array}{l}3+4 \\
\text { (GG2) }\end{array}$} & \multirow{2}{*}{$\begin{array}{l}4+3 \\
(G G 3)\end{array}$} & \multirow{2}{*}{$\begin{array}{l}8 \\
\text { (GG4) }\end{array}$} & \multirow{2}{*}{$\begin{array}{l}9-10 \\
\text { (GG5) }\end{array}$} & & & \\
\hline & & & & & & & Upgrade & No Change & Downgrade \\
\hline 6 (GGI) & 71 & $15(21.1)$ & $35(49.3)$ & II (I5.5) & $7(9.9)$ & $3(4.2)$ & $56(78.9)$ & $15(21.1)$ & - \\
\hline $3+4$ (GG2) & 40 & I (2.5) & $16(40.0)$ & $14(35.0)$ & $5(12.5)$ & $4(10.0)$ & $23(57.5)$ & $16(40.0)$ & I (2.5) \\
\hline $4+3$ (GG3) & 44 & $2(4.5)$ & $4(9.1)$ & $22(50.0)$ & $10(22.7)$ & $6(13.7)$ & $16(36.4)$ & $22(50.0)$ & $6(13.6)$ \\
\hline 8 (GG4) & 45 & I (2.2) & $I(2.2)$ & $7(15.6)$ & $24(53.3)$ & $12(26.7)$ & $12(26.7)$ & $24(53.3)$ & $9(20.0)$ \\
\hline 9-10 (GG5) & 22 & - & $\mathrm{I}(4.5)$ & - & $3(13.6)$ & $18(81.9)$ & - & 18 (81.9) & $4(18.1)$ \\
\hline Total & 222 & $19(8.6)$ & $57(25.7)$ & $54(24.3)$ & $49(22.1)$ & 43 (19.3) & $107(48.2)$ & $95(42.8)$ & $20(9.0)$ \\
\hline
\end{tabular}

Table 3 Multivariable Logistic Regression Analyses Predicting Extracapsular Extension in Patients Diagnosed with TRUS-Guided Biopsy and Treated with Radical Prostatectomy

\begin{tabular}{|c|c|c|c|c|c|c|}
\hline & \multicolumn{2}{|l|}{ Model I } & \multicolumn{2}{|l|}{ Model 2} & \multicolumn{2}{|l|}{ Model 3} \\
\hline & OR (95\% Cl) & $p$ value & OR $(95 \% \mathrm{Cl})$ & $p$ value & OR (95\% CI) & $p$ value \\
\hline TPSA & $1.02(1.01-1.04)$ & $<0.001$ & $1.03(1.01-1.04)$ & $<0.001$ & $1.01(1.00-1.02)$ & 0.049 \\
\hline CTZV & $1.02(1.001-1.04)$ & 0.04 & $1.02(1.001-1.04)$ & 0.038 & $1.01(0.99-1.04)$ & 0.355 \\
\hline \multicolumn{7}{|l|}{ PSAD } \\
\hline$\leq 0.20$ & I (Ref.) & & I (Ref.) & & I (Ref.) & \\
\hline$>0.20$ & $9.18(2.04-41.29)$ & 0.004 & $8.32(1.85-37.50)$ & 0.006 & $7.98(1.52-41.93)$ & 0.014 \\
\hline Maximum diameter of the index lesion at bp-MRI & & & $0.85(0.55-1.30)$ & 0.444 & $0.8 I(0.5 I-1.29)$ & 0.38 \\
\hline \multicolumn{7}{|l|}{ EPE at bp-MRI } \\
\hline No & & & I (Ref.) & & I (Ref.) & \\
\hline Yes & & & $2.93(1.27-6.74)$ & 0.012 & $2.45(0.98-6.09)$ & 0.054 \\
\hline \multicolumn{7}{|l|}{ SVI at bp-MRI } \\
\hline No & & & I (Ref.) & & I (Ref.) & \\
\hline Yes & & & $0.65(0.29-1.48)$ & 0.309 & $\mathrm{I} .03(0.4 \mathrm{I}-2.56)$ & 0.954 \\
\hline \multicolumn{7}{|l|}{ Biopsy grade group } \\
\hline I & & & & & I (Ref.) & \\
\hline 2 & & & & & $0.97(0.35-2.70)$ & 0.953 \\
\hline 3 & & & & & $1.75(0.63-4.84)$ & 0.284 \\
\hline 4 & & & & & $1.19(0.44-3.23)$ & 0.733 \\
\hline 5 & & & & & $8.39(1.55-45.29)$ & 0.013 \\
\hline No. of positive biopsy cores & & & & & $1.33(1.18-1.49)$ & $<0.001$ \\
\hline
\end{tabular}

patients had suspected SVI on bp-MRI. The median number of positive biopsy cores was 6 (IQR 3-11).

\section{Novel Models Predicting EPE, SVI, and Upgrading}

In multivariable analyses (Table 3 ), in model 1, TPSA (OR 1.015; 95\% CI: $1.006-1.02 ; P=0.001)$ and PSAD $(>0.20$ versus $\leq 0.20)(\mathrm{OR} 3.30 ; 95 \% \mathrm{CI}: 1.08-10.10 ; P=0.037)$ were significantly associated with EPE. In model 3 , only the biopsy GG (GG 5 versus GG 1) (OR 5.29; 95\% CI: $1.42-19.77 ; P=0.013$ ) and No. of positive biopsy cores (OR 1.32; 95\% CI: 1.18-1.48; $P<0.001$ ) were independent risk factors for EPE. In Figure 1A, despite the inclusion of bp-MRI findings, the AUC of model 2 was 0.763 (0.697-0.829), smaller than that of model 1 (AUC 0.766; 95\% CI: 0.701-0.831). Nonetheless, model 3 outperformed models 1 and 2 .

Table 4 depicts the results of multivariable analyses for SVI. We found that among the three clinical variables, 

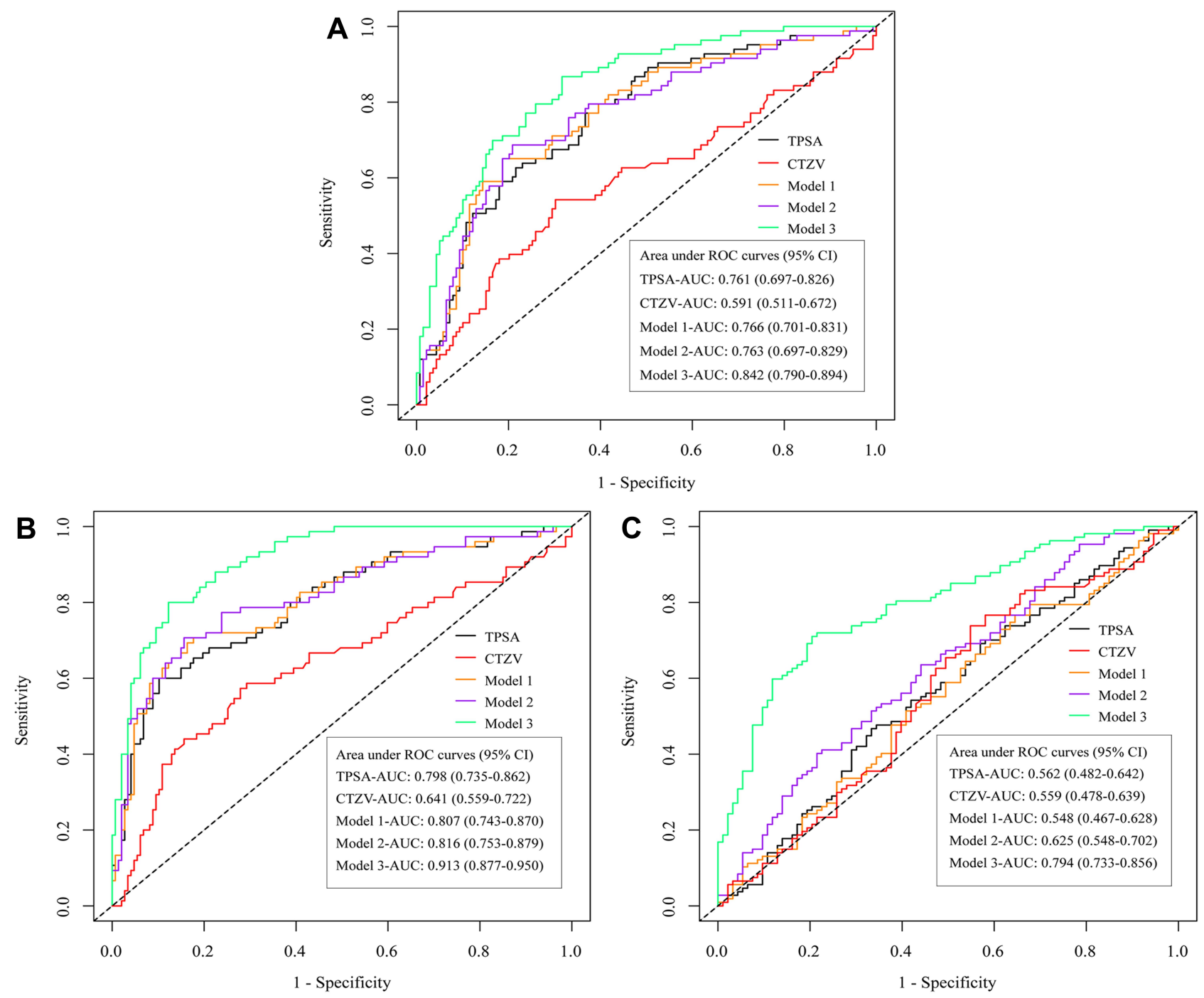

Figure I ROC curve analyses for the performance of models for the prediction of EPE (A), SVI (B), and upgrading (C).

only TPSA (OR $1.03 ; 95 \%$ CI: $1.02-1.05 ; P<0.001$ ) was significantly associated with presence of SVI at final pathology. Similarly, in model 2, only TPSA (OR 1.04; 95\% CI: $1.02-1.05 ; P<0.001)$ was significantly associated with SVI, whereas in model 3 , after the inclusion of biopsy data, TPSA (OR 1.02; 95\% CI: 1.01-1.03; $P=$ 0.004), biopsy GG4 (GG 4 versus GG 1) (OR 3.75; 95\% CI: $1.16-12.13 ; P=0.027)$, and the No. of positive biopsy cores (OR 1.52; 95\% CI: 1.31-1.76; $P<0.001$ ) were independent risk factors for SVI. As shown in Figure 1B, with the incremental addition of information on bp-MRI and TRUS-guided biopsy, the discrimination of models for predicting SVI improved significantly.

As for the results of multivariable analyses for upgrading after exclusion of patients with biopsy GG 5 (Table 5), we found that none of the three clinical variables had predictive value for upgrading in model $1(P>0.05)$, whereas in model 2 , after adding information on bp-MRI, the maximum diameter of the index lesion at bp-MRI (OR 0.65; 95\% CI: 0.44-0.96; $P=0.03$ ) and SVI at bp-MRI (Yes versus No) (OR 3.11; 95\% CI: $1.41-6.86 ; P=0.005)$ were significantly associated with upgrading at RP. In model 3, SVI at bp-MRI (Yes versus No) (OR 3.51; 95\% CI: 1.47-8.38; $P=0.005$ ), biopsy GG 2 (OR 0.27 ; 95\% CI: $0.10-0.69 ; P=0.006$ ), biopsy GG 3 (OR 0.10; 95\% CI: $0.04-0.25 ; P<0.001$ ) and biopsy GG 4 (OR 0.05; 95\% CI: $0.02-0.14 ; P<0.001)$ were significantly associated with upgrading. Among the models for predicting upgrading, model 3, which included clinical variables, information of bpMRI, and biopsy data, depicted the highest discrimination as compared with models 1 and 2 ([AUC 0.794; 95\% CI: 0.7330.856 ] vs [AUC $0.548 ; 95 \%$ CI: $0.467-0.628$ ] and [AUC 0.625; 95\% CI: 0.548-0.702]) (Figure 1C). 
Table 4 Multivariable Logistic Regression Analyses Predicting Seminal Vesicle Invasion in Patients Diagnosed with TRUS-Guided Biopsy and Treated with Radical Prostatectomy

\begin{tabular}{|c|c|c|c|c|c|c|}
\hline & \multicolumn{2}{|l|}{ Model I } & \multicolumn{2}{|l|}{ Model 2} & \multicolumn{2}{|l|}{ Model 3} \\
\hline & OR $(95 \% \mathrm{CI})$ & $p$ value & OR $(95 \% \mathrm{Cl})$ & $p$ value & OR $(95 \% \mathrm{CI})$ & $p$ value \\
\hline TPSA & $1.03(1.02-1.05)$ & $<0.001$ & $1.04(1.02-1.05)$ & $<0.001$ & $1.02(1.01-1.03)$ & 0.004 \\
\hline CTZV & $1.02(0.997-1.04)$ & 0.11 & $1.02(0.998-1.04)$ & 0.085 & $1.00(0.98-1.03)$ & 0.879 \\
\hline \multicolumn{7}{|l|}{ PSAD } \\
\hline$\leq 0.20$ & I (Ref.) & & I (Ref.) & & I (Ref.) & \\
\hline$>0.20$ & $1.66(0.52-5.29)$ & 0.392 & $1.48(0.46-4.75)$ & 0.514 & $0.58(0.14-2.45)$ & 0.463 \\
\hline Maximum diameter of the index lesion at bp-MRI & & & $0.83(0.52-1.31)$ & 0.417 & $0.68(0.40-1.17)$ & 0.16 \\
\hline \multicolumn{7}{|l|}{ EPE at bp-MRI } \\
\hline No & & & I (Ref.) & & I (Ref.) & \\
\hline Yes & & & $2.37(0.994-5.65)$ & 0.051 & $1.99(0.69-5.74)$ & 0.202 \\
\hline \multicolumn{7}{|l|}{ SVI at bp-MRI } \\
\hline No & & & I (Ref.) & & I (Ref.) & \\
\hline Yes & & & $0.63(0.26-\mid .5 I)$ & 0.302 & $1.29(0.43-3.86)$ & 0.644 \\
\hline \multicolumn{7}{|l|}{ Biopsy grade group } \\
\hline I & & & & & I (Ref.) & \\
\hline 2 & & & & & $1.06(0.29-3.86)$ & 0.935 \\
\hline 3 & & & & & $1.93(0.57-6.49)$ & 0.289 \\
\hline 4 & & & & & $3.75(1.16-12.13)$ & 0.027 \\
\hline 5 & & & & & $3.76(0.93-15.24)$ & 0.064 \\
\hline No. of positive biopsy cores & & & & & $1.52(1.31-1.76)$ & $<0.001$ \\
\hline
\end{tabular}

In Figure 2, for predicting adverse pathological features, model 3 always showed the highest net benefit compared with models 1 and 2. Furthermore, the novel three risk calculators were created on the basis of the results of model 3 (an online tool is provided in the Supplementary Material).

\section{Discussion}

Prostate cancer is highly prevalent worldwide, and more and more patients are suffering from this type of malignancy. ${ }^{1,2}$ Due to the paucity of knowledge of $\mathrm{PCa}$ patients at high risk of harboring adverse pathology, tailoring the best therapy to patients represents a great challenge. Predictive models which could identify patients harboring adverse pathological characteristics such as EPE, SVI, and upgrading before RP are extremely important in the treatment planning and decision-making processes. ${ }^{5,6,11}$ Previous studies suggested that the inclusion of information derived from mp-MRI and MRI-targeted biopsies could improve the discrimination of predictive models. ${ }^{7,11,12,20,21}$ However, mp-MRI requires a long acquisition time and additional cost.
Models for predicting adverse pathological features based on readily available variables are scarce. Therefore, in the present study, we evaluated whether the addition of information obtained by bp-MRI and TRUS-guided systematic needle biopsy, which are easily applied in clinical practice, would improve the ability of those predictive models to identify EPE, SVI, and upgrading before RP.

In the present study, we first introduced the variables of CTZV and global biopsy Gleason grade group in multivariable analyses. Porcaro and colleagues ${ }^{22-24}$ demonstrated that CTZV and CTZV-based prostate volume index were significantly associated with the tumor load of PCa. The enlargement of the prostate central transitional zone resulted in prostate enlargement. Variations in volume between central transitional zones and peripheral prostate zones reflect different responses to systemic and local conditions. ${ }^{22}$ In addition, considering that the global biopsy GG rather than the highest biopsy GG is more likely to be in line with RP GG, we then selected global GG together with other preoperative clinical parameters to construct predictive models to calculate the probability of adverse pathological features for PCa patients. ${ }^{25}$ 
Table 5 Multivariable Logistic Regression Analyses Predicting Upgrading in Patients Diagnosed with TRUS-Guided Biopsy and Treated with Radical Prostatectomy

\begin{tabular}{|c|c|c|c|c|c|c|}
\hline & \multicolumn{2}{|l|}{ Model I } & \multicolumn{2}{|l|}{ Model 2} & \multicolumn{2}{|l|}{ Model 3} \\
\hline & OR (95\% Cl) & $p$ value & OR (95\% CI) & $p$ value & OR (95\% CI) & $p$ value \\
\hline TPSA & $1.001(0.995-1.01)$ & 0.656 & $1.002(0.995-1.01)$ & 0.65 & $1.003(0.995-1.01)$ & 0.485 \\
\hline CTZV & $1.004(0.99-1.02)$ & 0.682 & I.0I (0.99-I.03) & 0.429 & I.0I (0.99-I.04) & 0.22 \\
\hline \multicolumn{7}{|l|}{ PSAD } \\
\hline$\leq 0.20$ & I (Ref.) & & I (Ref.) & & I (Ref.) & \\
\hline$>0.20$ & I.I2(0.5I-2.46) & 0.772 & $1.16(0.52-2.6 \mathrm{I})$ & 0.722 & $2.00(0.77-5.19)$ & 0.154 \\
\hline $\begin{array}{l}\text { Maximum diameter of the index lesion at bp- } \\
\text { MRI }\end{array}$ & & & $0.65(0.44-0.96)$ & 0.03 & $0.70(0.45-1.09)$ & 0.113 \\
\hline \multicolumn{7}{|l|}{ EPE at bp-MRI } \\
\hline No & & & I (Ref.) & & I (Ref.) & \\
\hline Yes & & & $0.61(0.29-1.30)$ & 0.199 & $0.58(0.25-1.33)$ & 0.197 \\
\hline \multicolumn{7}{|l|}{ SVI at bp-MRI } \\
\hline No & & & I (Ref.) & & I (Ref.) & \\
\hline Yes & & & $3.11(1.4 I-6.86)$ & 0.005 & $3.51(1.47-8.38)$ & 0.005 \\
\hline \multicolumn{7}{|l|}{ Biopsy grade group } \\
\hline I & & & & & I (Ref.) & \\
\hline 2 & & & & & $0.27(0.10-0.69)$ & 0.006 \\
\hline 3 & & & & & $0.10(0.04-0.25)$ & $<0.001$ \\
\hline 4 & & & & & $0.05(0.02-0.14)$ & $<0.001$ \\
\hline No. of positive biopsy cores & & & & & $1.09(0.98-1.2 \mathrm{I})$ & 0.136 \\
\hline
\end{tabular}

Our study has several important findings. First, although we did not include mp-MRI information in our study, the addition of bp-MRI still improved the discrimination of models for predicting SVI and upgrading. As seen in ROC analyses (Figure 1), except for the model for predicting EPE, all models based on clinical variables and bp-MRI had higher AUC values than the corresponding models based on clinical variables alone ( 0.816 vs 0.807 for SVI and 0.625 vs 0.548 for upgrading). Information on bp-MRI had low sensitivity for predicting EPE, and this was in line with the findings of a previous study. ${ }^{26} \mathrm{In}$ contrast to our series, Gupta et $\mathrm{al}^{14}$ demonstrated that mpMRI alone performs better than Partin tables in the prediction of EPE. Moreover, for the prediction of EPE, Gandaglia et $\mathrm{al}^{11}$ showed that the inclusion of clinical stage at mp-MRI and maximum diameter of the index lesion at mp-MRI achieved a higher AUC value than the model based on clinical covariates alone ( 0.70 vs 0.67$)$. Similarly, Rayn et $\mathrm{al}^{7}$ reported that when mp-MRI findings were added to the systematic biopsy-based MSKCC nomogram, the AUC increased by 0.10 for predicting EPE. These results indicated that dynamic contrast- enhanced imaging may play a pivotal role in tumor local staging for PCa.

Second, compared with models 1 and 2, model 3, which incorporated clinical variables, bp-MRI findings, and TRUSguided systematic needle biopsies, significantly improved the discrimination for predicting adverse pathological features. Similar to our results, nomograms conducted by Tosco et $\mathrm{al}^{27}$ based on clinical variables and systematic biopsy information suggested good performance for prediction of EPE (AUC 0.77; 95\% CI: 0.76-0.79) and SVI (AUC 0.82; 95\% CI: 0.79-0.85). Although the inclusion of bp-MRI did not improve discrimination in the prediction of EPE, the addition of biopsy data achieved the highest AUC of 0.842 (95\% CI: 0.790-0.894). Thus, model 3 for predicting EPE could help clinicians to select appropriate candidates suitable for receiving neurovascular bundle-sparing surgery. Notwithstanding better performance of MRI-targeted biopsies in detecting clinically significant $\mathrm{PCa}$ and reflecting aggressive behavior of PCa, TRUS-guided systematic needle biopsies may be non-inferior to it in predicting adverse pathology at RP. ${ }^{11,28}$ The added value of systematic biopsy data in our study may be owing to the multifocal nature of 

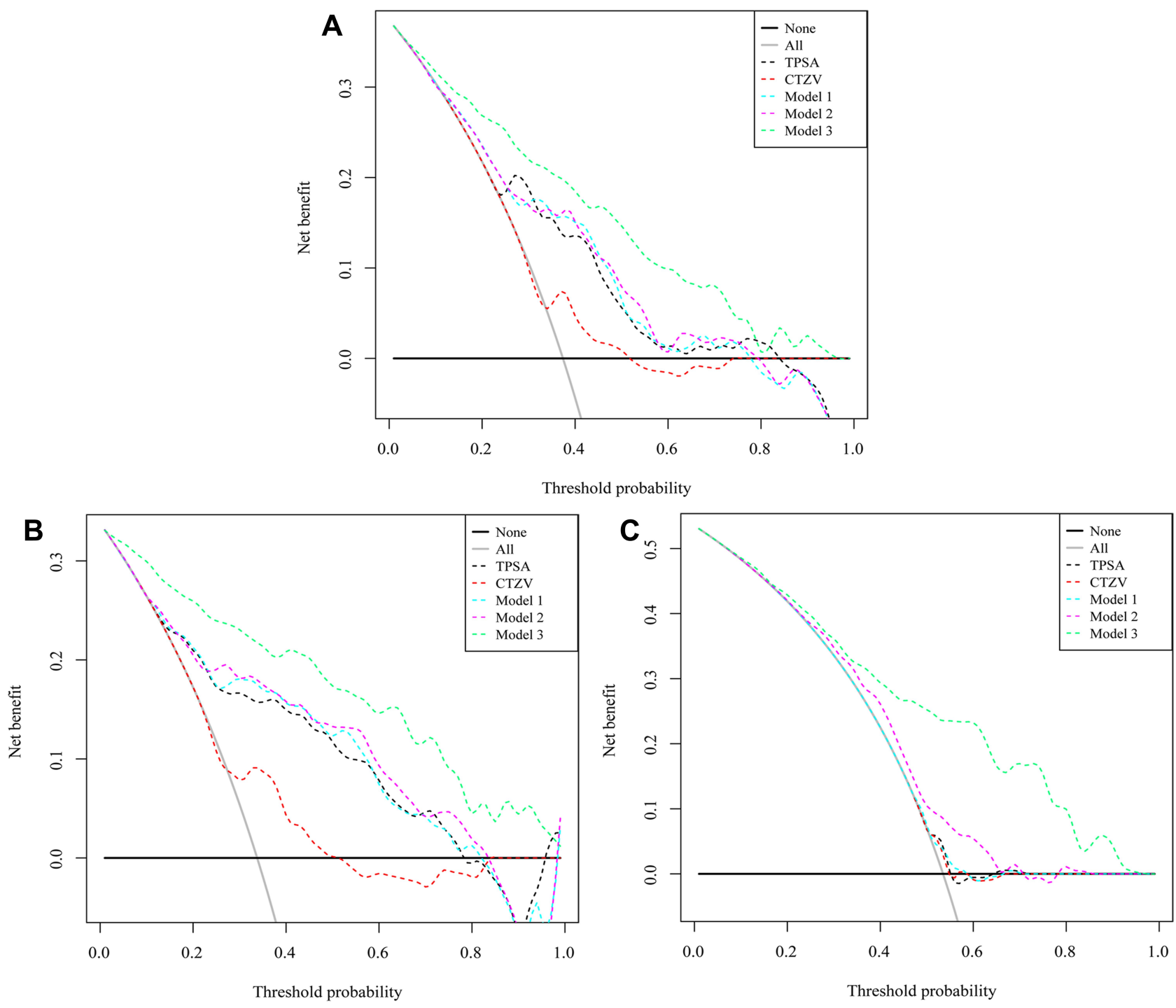

Figure 2 Decision-curve analyses demonstrating the net benefit associated with the use of the novel risk calculators for the prediction of EPE (A), SVI (B), and upgrading (C).

PCa. ${ }^{29,30}$ With regard to the results of MRI-targeted biopsy, the existence of multiple tumor foci might lead to underestimation of the risk of harboring adverse pathological characteristics. Additionally, the number of positive cores was independently associated with EPE and SVI, and this may be related to the fact that this variable could be considered as the proxy of tumor volume. ${ }^{31}$ In particular, biopsy GG 1 patients would more likely upgrade to a higher GG at RP, followed by biopsy GG 2, biopsy GG 3, and biopsy GG 4. Trpkov et $\mathrm{al}^{25}$ also reported similar findings. These observations explain why some patients with GG 1 disease at biopsy suffer metastases or die of prostate cancer and suggest that a considerable proportion of biopsy GG 1 patients who embark on active surveillance are not, in fact, suitable candidates. $^{32}$
We must acknowledge that mp-MRI- and MRI-targeted biopsy systems represent relatively expensive tools, and they are not available in every hospital. Our novel risk calculators are based on readily available variables such as bp-MRI and systematic biopsy data and are clinically useful in identifying those PCa patients with adverse pathological features. By applying our risk calculators, it would be easier for clinicians to make decisions regarding tailoring individualized therapy to each patient. For example, clinicians would not perform nerve-sparing surgery for a patient with a high probability of EPE calculated by the EPE risk calculator.

Despite several strengths, our study has certain limitations. First, the data on PCa patients who underwent RP enrolled in our study cohort were retrospectively collected at a single institution, and this may have resulted in 
a selection bias. Second, the cohort was not representative of all Chinese PCa patients, and a formal external validation is needed. Finally, the case-level highest Gleason grade group was more commonly assigned to patients undergoing systematic TRUS-guided biopsy in our country; hence, we should also construct predictive models to identify risk factors associated with upgrading under the comparison between the highest biopsy GG and final RP samples.

\section{Conclusions}

In summary, we developed three novel risk calculators with relatively good discrimination and accuracy to help clinicians identify the individualized risk of EPE, SVI, or upgrading for PCa patients after prostate needle biopsy. Those readily available variables of bp-MRI and TRUSguided systematic biopsy could improve the discrimination of models for predicting adverse pathological features. With an estimate of individual risk, more accurate risk stratification can be assigned to patients. Clinicians and patients can then make more appropriate treatment decisions. Of course, further external validation of the three risk calculators is necessary.

\section{Author Contributions}

All authors contributed to data analysis, drafting or revising the article, have agreed on the journal to which the article will be submitted, gave final approval of the version to be published, and agree to be accountable for all aspects of the work.

\section{Disclosure}

The authors report no conflicts of interest for this work.

\section{References}

1. Ferlay J, Colombet M, Soerjomataram I, et al. Estimating the global cancer incidence and mortality in 2018: globocan sources and methods. Int J Cancer. 2019;144(8):1941-1953. doi:10.1002/ijc.31937

2. Siegel RL, Miller KD, Jemal A. Cancer statistics, 2019. CA Cancer J Clin. 2019;69(1):7-34. doi:10.3322/caac.21551

3. Chen W, Zheng R, Baade PD, et al. Cancer statistics in china, 2015. CA Cancer J Clin. 2016;66(2):115-132. doi:10.3322/caac.21338

4. Preisser F, Marchioni M, Nazzani S, et al. The impact of lymph node metastases burden at radical prostatectomy. Eur Urol Focus. 2019;5 (3):399-406. doi:10.1016/j.euf.2017.12.009

5. Memorial sloan kettering cancer center. Prediction tools/prostate cancer nomograms: pre- radical prostatectomy. Memorial sloan kettering cancer center (MSKCC); 2018. Available from: https://www.mskcc. org/nomograms/prostate/pre_op. Accessed August 11, 2020.

6. Tosoian JJ, Chappidi M, Feng Z, et al. Prediction of pathological stage based on clinical stage, serum prostate-specific antigen, and biopsy gleason score: partin tables in the contemporary era. BJU Int. 2017;119(5):676-683. doi:10.1111/bju.13573
7. Rayn KN, Bloom JB, Gold SA, et al. Added value of multiparametric magnetic resonance imaging to clinical nomograms for predicting adverse pathology in prostate cancer. $J$ Urol. 2018;200 (5):1041-1047. doi:10.1016/j.juro.2018.05.094

8. Park BH, Jeon HG, Jeong BC, et al. Influence of magnetic resonance imaging in the decision to preserve or resect neurovascular bundles at robotic assisted laparoscopic radical prostatectomy. J Urol. 2014;192 (1):82-88. doi:10.1016/j.juro.2014.01.005

9. Ueno Y, Tamada T, Bist V, et al. Multiparametric magnetic resonance imaging: current role in prostate cancer management. Int $J$ Urol. 2016;23(7):550-557. doi:10.1111/iju.13119

10. Feng TS, Sharif-Afshar AR, Wu J, et al. Multiparametric MRI improves accuracy of clinical nomograms for predicting extracapsular extension of prostate cancer. Urology. 2015;86(2):332-337. doi:10.1016/j.urology.2015.06.003

11. Gandaglia G, Ploussard G, Valerio M, et al. The key combined value of multiparametric magnetic resonance imaging, and magnetic resonance imaging-targeted and concomitant systematic biopsies for the prediction of adverse pathological features in prostate cancer patients undergoing radical prostatectomy. Eur Urol. 2019;77 (6):733-741. doi:10.1016/j.eururo.2019.09.005

12. Martini A, Gupta A, Lewis SC, et al. Development and internal validation of a side-specific, multiparametric magnetic resonance imaging-based nomogram for the prediction of extracapsular extension of prostate cancer. BJU Int. 2018;122(6):1025-1033. doi:10.1111/bju.14353

13. Boesen L, Thomsen FB, Norgaard N, et al. A predictive model based on biparametric magnetic resonance imaging and clinical parameters for improved risk assessment and selection of biopsy-naive men for prostate biopsies. Prostate Cancer Prostatic Dis. 2019;22 (4):609-616. doi:10.1038/s41391-019-0149-y

14. Gupta RT, Faridi KF, Singh AA, et al. Comparing 3-T multiparametric MRI and the partin tables to predict organ-confined prostate cancer after radical prostatectomy. Urol Oncol. 2014;32(8):1292-1299. doi:10.1016/j.urolonc.2014.04.017

15. Xu L, Zhang G, Shi B, et al. Comparison of biparametric and multiparametric MRI in the diagnosis of prostate cancer. Cancer Imaging. 2019;19(1):90. doi:10.1186/s40644-019-0274-9

16. Weinreb JC, Barentsz JO, Choyke PL, et al. PI-RADS prostate imaging - reporting and data system: 2015, version 2. Eur Urol. 2016;69(1):16-40. doi:10.1016/j.eururo.2015.08.052

17. Barentsz JO, Weinreb JC, Verma S, et al. Synopsis of the PI-RADS v2 guidelines for multiparametric prostate magnetic resonance imaging and recommendations for use. Eur Urol. 2016;69(1):41-49. doi:10.1016/j.eururo.2015.08.038

18. Epstein JI, Zelefsky MJ, Sjoberg DD, et al. A contemporary prostate cancer grading system: a validated alternative to the gleason score. Eur Urol. 2016;69(3):428-435. doi:10.1016/j.eururo.2015. 06.046

19. Vickers AJ, Cronin AM, Elkin EB, et al. Extensions to decision curve analysis, a novel method for evaluating diagnostic tests, prediction models and molecular markers. BMC Med Inform Decis Mak. 2008;8 (1):53. doi:10.1186/1472-6947-8-53

20. Morlacco A, Sharma V, Viers BR, et al. The incremental role of magnetic resonance imaging for prostate cancer staging before radical prostatectomy. Eur Urol. 2017;71(5):701-704. doi:10.1016/j. eururo.2016.08.015

21. Tay KJ, Gupta RT, Brown AF, et al. Defining the incremental utility of prostate multiparametric magnetic resonance imaging at standard and specialized read in predicting extracapsular extension of prostate cancer. Eur Urol. 2016;70(2):211-213. doi:10.1016/j.eururo.2015. 10.041

22. Porcaro AB, Tafuri A, Sebben M, et al. Prostate volume index and prostatic chronic inflammation predicted low tumor load in 945 patients at baseline prostate biopsy. World J Urol. 2020;38 (4):957-964. doi:10.1007/s00345-019-02830-7 
23. Porcaro AB, Tafuri A, Sebben M, et al. Prostate volume index is able to differentiate between prostatic chronic inflammation and prostate cancer in patients with normal digital rectal examination and prostate-specific antigen values $<10 \mathrm{ng} / \mathrm{mL}$ : results of 564 biopsy naive cases. Urol Int. 2019;103(4):415-422. doi:10.1159/000502659

24. Porcaro AB, Tafuri A, Sebben M, et al. Prostate volume index and prostatic chronic inflammation have an effect on tumor load at baseline random biopsies in patients with normal DRE and PSA values less than $10 \mathrm{ng} / \mathrm{mL}$ : results of 564 consecutive cases. Ther Adv Urol. 2019;11:1756287219868604. doi:10.1177/1756287219868604

25. Trpkov K, Sangkhamanon S, Yilmaz A, et al. Concordance of "case level" global, highest, and largest volume cancer grade group on needle biopsy versus grade group on radical prostatectomy. Am J Surg Pathol. 2018;42(11):1522-1529. doi:10.1097/PAS.000000 0000001137

26. de Rooij M, Hamoen EH, Witjes JA, et al. Accuracy of magnetic resonance imaging for local staging of prostate cancer: a diagnostic meta-analysis. Eur Urol. 2016;70(2):233-245. doi:10.1016/j. eururo.2015.07.029

27. Tosco L, De Coster G, Roumeguere T, et al. Development and external validation of nomograms to predict adverse pathological characteristics after robotic prostatectomy: results of a prospective, multi-institutional, nationwide series. Eur Urol Oncol. 2018;1 (4):338-345. doi:10.1016/j.euo.2018.04.008
28. Kasivisvanathan V, Rannikko AS, Borghi M, et al. MRI-targeted or standard biopsy for prostate-cancer diagnosis. $N$ Engl J Med. 2018;378(19):1767-1777. doi:10.1056/NEJMoa1801993

29. Alchin DR, Murphy D, Lawrentschuk N. Risk factors for gleason score upgrading following radical prostatectomy. Minerva Urol Nefrol. 2017;69(5):459-465. doi:10.23736/S0393-2249.16.02684-9

30. Johnson DC, Raman SS, Mirak SA, et al. Detection of individual prostate cancer foci via multiparametric magnetic resonance imaging. Eur Urol. 2019;75(5):712-720. doi:10.1016/j.eururo.20 18.11.031

31. Gandaglia G, Ploussard G, Valerio M, et al. A novel nomogram to identify candidates for extended pelvic lymph node dissection among patients with clinically localized prostate cancer diagnosed with magnetic resonance imaging-targeted and systematic biopsies. Eur Urol. 2019;75(3):506-514. doi:10.1016/j.eururo.2018.10.012

32. Yang DD, Mahal BA, Muralidhar V, et al. Pathologic outcomes of gleason 6 favorable intermediate-risk prostate cancer treated with radical prostatectomy: implications for active surveillance. Clin Genitourin Cancer. 2018;16(3):226-234. doi:10.1016/j.clgc.2017.10. 013

\section{Publish your work in this journal}

Cancer Management and Research is an international, peer-reviewed open access journal focusing on cancer research and the optimal use of preventative and integrated treatment interventions to achieve improved outcomes, enhanced survival and quality of life for the cancer patient.
The manuscript management system is completely online and includes a very quick and fair peer-review system, which is all easy to use. Visit http://www.dovepress.com/testimonials.php to read real quotes from published authors. 